\begin{tabular}{|c|l|}
\hline Title & Laser micromachining of porous anodic al umina film \\
\hline Author(s) & Jha, Himendra; Kikuchi, Tatsuya; Sakairi, Masatoshi; T akahashi, Hideaki \\
\hline Citation & $\begin{array}{l}\text { A pplied Physics A: Materials Science \& Processing, 88(4), 617-622 } \\
\text { https://doi.org/40.1007/300339-007-4140-4 }\end{array}$ \\
\hline Issue Date & 2007-09 \\
\hline Doc URL & http://hdl.handle.net/2115/28634 \\
\hline Rights & The original publication is available at www.springerlink.com \\
\hline Type & article (author version) \\
\hline File Information & APA 88-4.pdf \\
\hline
\end{tabular}

Instructions for use 


\title{
Laser micromachining of porous anodic alumina film
}

\author{
Himendra Jha, Tatsuya Kikuchi, Masatoshi Sakairi, and Hideaki Takahashi* \\ Lab. of Interface Microstructure Analysis (LIMSA) \\ Division of Materials Science and Engineering, Graduate School of Engineering, \\ Hokkaido University, Kita-13 Nishi-8, Kita-Ku, Sapporo 060-8628, Japan
}

*Corresponding author: Prof. Hideaki Takahashi

Tel: $+81-11-706-7110$

Fax: + 81-11-706-7881

E-mail:takahasi@elechem1-mc.eng.hokudai.ac.jp

\begin{abstract}
A laser direct-write process on porous anodic alumina film is carried out for the fabrication of microstructure on the film, using nanosecond, second harmonic Nd:YAG laser. Laser micromachining can be preformed in two different ways on colored and pore-sealed anodic alumina film, to generate microstructures on the film. Removal of the aluminum substrate before laser irradiation greatly improves the shape characteristics of the microstructure. The depth of microstructures can be controlled by the power and the scanning speed of the laser beam. Several structures with depths from 1 to $35 \mu \mathrm{m}$ were fabricated on anodic alumina film with good precision and reproducibility.
\end{abstract}

PACS 79.20.Ds; 82.45.Cc; 42.62.Cf

\section{Introduction}

Micromachining is an important tool in the rapidly growing micro-technology industries. Micromachining of the surface and bulk of a material is widely applied for the fabrication of a wide variety of micro and nano-devices. Techniques like photolithography, LIGA, wet and dry etching, electron and photon beams application, and others are industrially well established [1-4]. Owing to the simplicity, flexibility and cost effectiveness, laser material processing is becoming increasingly popular over the last decade [5-7]. Laser processing is generally carried out using two basic approaches, laser direct writing or mask projection. In direct writing techniques, a laser beam is focused on a small spot at the surface of a material, and structures are made by moving either the laser spot or substrate in defined directions. This method is best suited for the generation of three-dimensional structures [8]. In the mask projection method, the laser beam is projected through a pre-designed photo-mask onto the surface of a material [9].

Silicon is a widely accepted material for the fabrication of the majority of microdevices, in almost all branches of technology, from medical to spacecraft applications. However, a number of other materials, such as polymers, glasses, ceramics, composite materials and metals have been widely investigated for their specific properties and applications. Porous anodic oxide film formed on aluminum by anodizing in oxalic acid solution is very similar to leuco-sapphire or glass ceramics, which are widely used in microelectronics. It also has sufficient mechanical strength and stability in extreme environments making it suitable for the subsequent fabrication of hybrid integrated circuits [10]. Recently, thick porous anodic oxide film has been explored as a promising material for the fabrication and design of various micro components and structures [11-13]. In this context, fabrication of microstructures on anodic alumina film, with a simple, precise, and cost effective method is of interest to industrial as well as research processes. 
A recent study [14] demonstrated that, coloring of the porous layer of the anodic oxide film on aluminum substrate with organic dyes followed by hydrothermal pore-sealing strongly enhances the light absorption properties of the film. This increase in absorption makes it possible to patterning of the film surface by nanosecond-visible laser beams. However, a fraction of the laser beam, which passes through the anodic oxide film, during laser irradiation, significantly damages the aluminum substrate near the interface with the film. The damage at the interface makes it impossible to use the higher powered laser beams or low scanning speeds that are required for deeper structures on the film surface. The microstructures formed in the anodic oxide film on aluminum also contain a large number of irregular shock defects at the edges of the structures. The reflection of a part of the laser beam from the film-metal interface may be responsible for such defects in the microstructures. Moreover, the presence of aluminum, attached to the film, limits applications to designs not involving elevated temperatures, as the melting point of aluminum is much lower than the anodic oxide film. The present research, found that removal of the aluminum substrate before laser irradiation overcomes these drawbacks, and offers a step forward in improving laser micromachining techniques for the fabrication of microstructures on anodic alumina film.

The present paper describes micromachining techniques for porous anodic alumina film (after removal from the aluminum substrate) with a nanosecond Nd:YAG laser beam. Different modes of laser ablation on the anodic alumina surface are explained. Similarly, fabrication of microstructures on anodic alumina film, and the effect of laser power and laser scanning speed on the shape and size of the microstructure are presented.

\section{Experimental Method}

\subsection{Preparation of anodic alumina film}

Porous anodic oxide film was formed on the aluminum substrate by anodizing electropolished specimens in $0.22 \mathrm{M} \mathrm{H}_{2} \mathrm{C}_{2} \mathrm{O}_{4}$ solution with a constant current of $100 \mathrm{Am}^{-2}$ at $293 \mathrm{~K}$. The thickness of the anodic alumina film formed was about $32 \mu \mathrm{m}$ and $64 \mu \mathrm{m}$ after anodizing for 2 and 4 hours respectively. Then the specimens were immersed in $0.029 \mathrm{M}$ Alizarin red S dyeing solution, for $300 \mathrm{~s}$ at $333 \mathrm{~K}$, to impregnate the dye in the pores of the anodic oxide film. The colored specimens were immersed in boiling doubly distilled water for $20 \mathrm{~min}$ for hydrothermal pore-sealing. Finally, the anodic alumina film was removed from the aluminum substrate either by amalgamating the aluminum in saturated $\mathrm{HgCl}_{2}$ solution or dissolving the aluminum in saturated $\mathrm{SnCl}_{4}$ solution at room temperature. The various steps in the la-

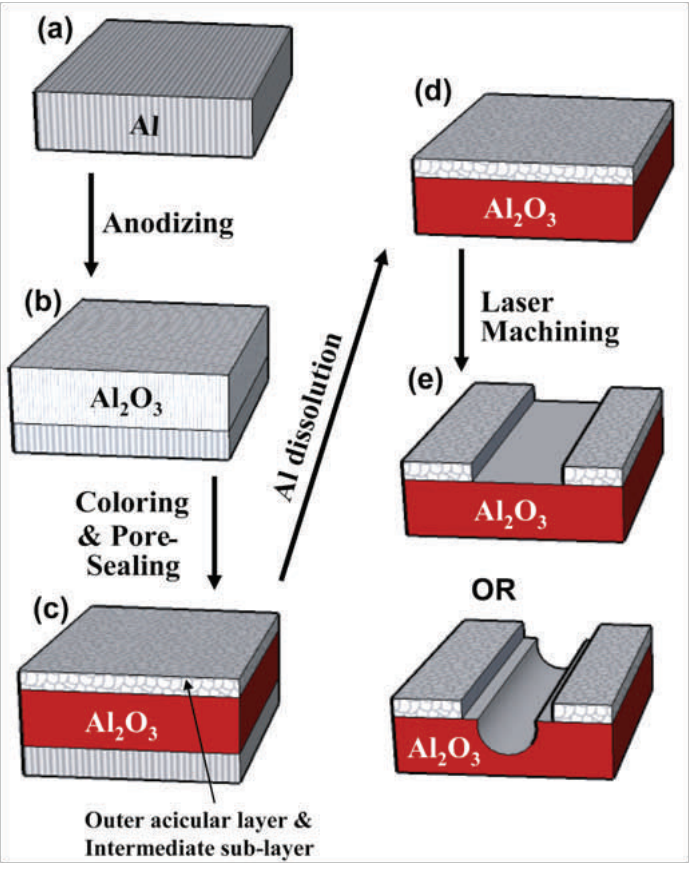

Figure 1: Schematic outline of the different steps in the laser micromachining on the anodic alumina film. (Not to scale) ser micromachining of anodic alumina film are summarized in Fig.1.

\subsection{Laser micromachining}

The anodic alumina film was mounted over a rigid frame for mechanical support and then the film was irradiated with a Q-switched, second harmonic, pulsed Nd:YAG laser (Spectra Physics; GCR-100 series) with $532 \mathrm{~nm}$ wavelength, $8 \mathrm{~ns}$ pulse width, and $50 \mathrm{~Hz}$ frequency. The laser irradiation was carried out inside a plastic cell filled with doubly distilled water. There was a quartz window in the cell for the passage of the laser beam, as shown in Fig. 2. The speed and direction of the 3D movable stage (KOHZU; PK 569-B) was varied in two dimensions, controlled by a computer.

Analysis of the laser irradiated part and microstructures on the anodic alumina film were carried out by confocal scanning laser microscopy, CSLM (Lasertec, 1LM21D) and field emission scanning electron microscopy, FE-SEM (JEOL, JSM-6500F). 


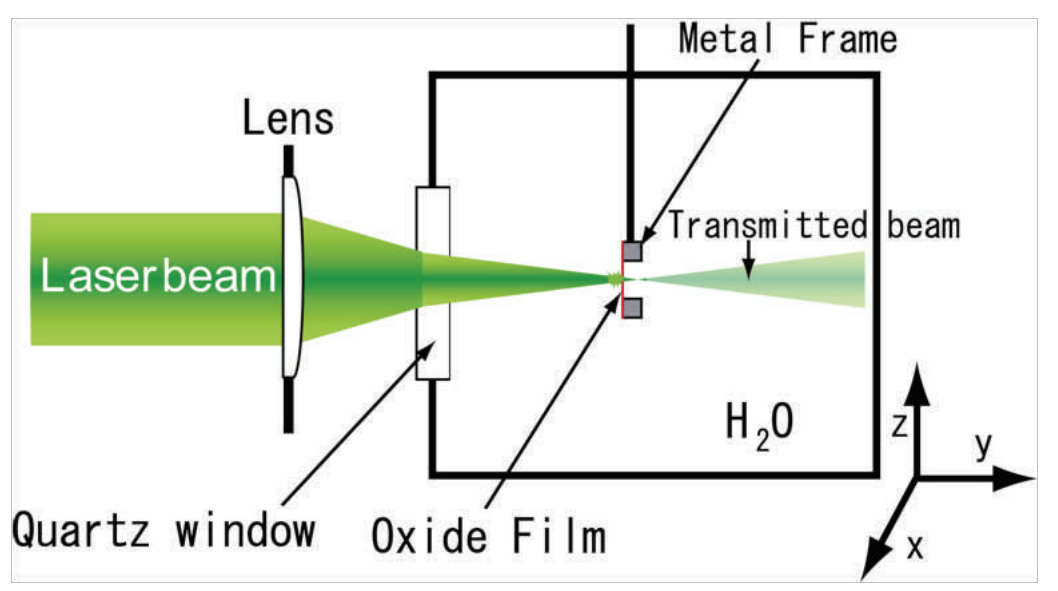

Figure 2: Laser micromachining set-up for the fabrication of microstructures on anodic alumina film.

\section{Results and discussion}

\subsection{Modes of laser-machining of anodic alumina film}

Figure 3 shows the FE-SEM images of laser micromachined areas on the anodic alumina. Fig. 3 (a) shows the surface of the as formed porous anodic alumina film, before pore-sealing. Numerous pores of a few tens of nanometers in diameter are clearly seen on the surface of the film. Coloring the porous film followed by laser micromachining results in a highly irregular and non-continuous structure on the anodic alumina film, as shown in Fig 3 (b). The magnified view of a part of the laser machined surface is shown in Fig. 3 (c), here the laser ablated part as well as intact porous film surface can be clearly identified. The laser ablated part shows the appearance of melted anodic alumina, suggesting local heating to melting by the irradiation of the $6 \mathrm{~mW}$ laser beam. Colored and hydro-thermally pore-sealed anodic alumina film forms a highly crystalline, acicular bhoemite layer on the film surface, as shown in Fig. 3 (d). Such a colored and pore-sealed anodic alumina surface allows the two types of laser micromachined structures on the film surface, as shown in Fig. 3 (e) and (f). Figure 3 (e) shows a deep groove on the film surface formed by ablating the bulk of the film by using a $6 \mathrm{~mW}$ laser beam. The figure shows that the top surface layer is randomly removed from the surface along the machined area. The inner part of the laser ablated area clearly shows the melting of the bulk anodic alumina film, as shown in Fig. 3 (g). On the other hand, low power laser beam machining on the colored and pore-sealed film results in the much less deep groove on the surface, as shown in Fig. 3 (f), which was fabricated by using a $4 \mathrm{~mW}$ laser beam. The magnified view of a part of the laser machined area shows that the highly crystalline bhoemite layer as well as a further layer (intermediate sub-layer) is removed evenly from the film surface, leaving a clean laser ablated area, as shown in Fig. 3 (h). Here, unlike in the deeper structure, the laser machined area does not show signs of melting.

The anodic alumina film, formed by anodizing of aluminum in oxalic acid solution is transparent and is light yellow in appearance. Due to the transparency of the anodic alumina film, it is very difficult to machine the film by the laser beam, as a higher fraction of the laser beam passes through the film. Therefore, coloring the film with organic dyes followed by pore-sealing enhances the light absorption properties of the film [14]. This increase in absorption makes it possible to machine the anodic alumina film on the aluminum substrate. However, it was observed that either coloring or pore-sealing of the film, separately, was not a sufficient condition for the laser machining of the film. Laser machining of the colored but not pore-sealed film results in discontinuous laser ablation at the laser irradiated surface, like in Fig. 3 (b). This shows the value of the outer acicular bhoemite layer and compact intermediate sub-layer formed on the film surface by the hydrothermal pore-sealing [15,16]. The layers formed on the surface can be precisely removed from the porous layer by the low power laser beam. Here, even irradiation with a low power laser beam, about 2 to $4 \mathrm{~mW}$, is sufficient to remove these two outermost layers from the film surface, as in Fig. 3 (f). The structure formed by such low power laser machining always has an almost constant depth of about 1 to $1.2 \mu \mathrm{m}$. However, increasing the laser power density, in the case of colored and pore-sealed film, not only the outer layers but also the bulk of the anodic alumina film is ablated from the film. The microstructure formed in this case has good edge definition and the ablation is taking place by melting of the anodic alumina at the laser irradiated area, as in Fig. 3 (g). 

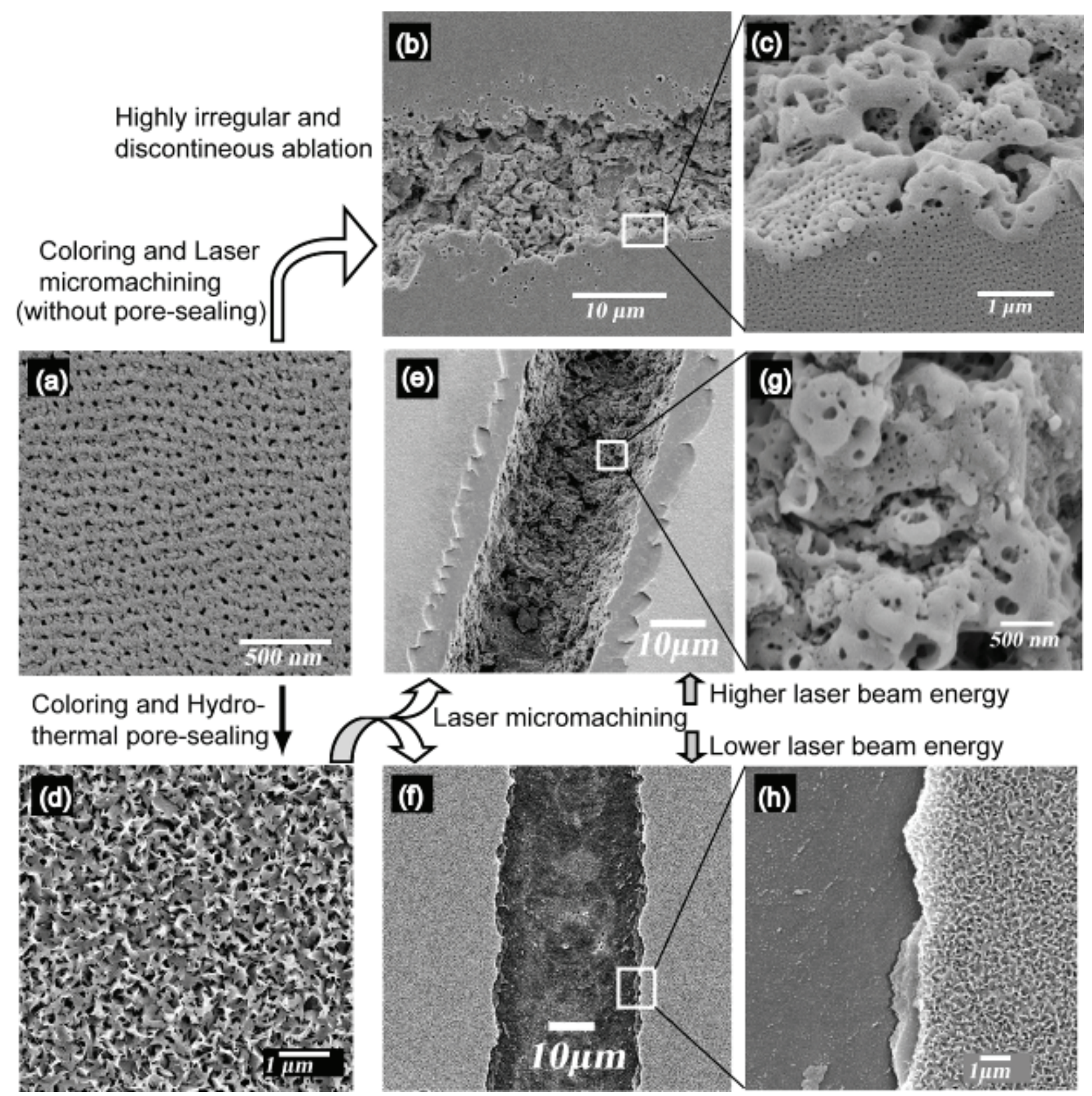

Figure 3: Mode of laser assisted removal from the anodic alumina film. (a) is the as formed porous anodic alumina film and, (d) after coloring and pore sealing. (b) and (c) laser micromachined film after coloring. (e) and (g) micromachining by higher laser power beam . (f) and (h) micromachining by lower laser power beam.

From the above discussion, it is clear that, the surface layer formed on the film surface during hydrothermal pore-sealing can be removed by applying a low power laser beam, resulting in an about 1 to $1.2 \mu \mathrm{m}$ deep groove on the film. The removal of the surface layers may be due to the thermal shock originating from the laser beam irradiation on the surface. Different from removing just the surface layers, in the machining of the bulk anodic alumina film, the irradiated laser energy must be sufficiently high to melt and vaporize the laser irradiated area. A number of models have been proposed and demonstrated for the ablation of materials by laser irradiation in the past few decades [17-20]. In general, it has been reported that nanosecond laser ablation of dielectrics is due to multi-photon absorption followed by an avalanche process. The absorbed laser energy first heats the target surface to the melting point and then to the vaporization temperature [20]. However the detailed mechanism of the laser ablation is difficult to specify because of the presence of organic dye as well as amorphous and crystalline forms of anodic alumina at the laser irradiated area.

\subsection{Fabrication of microstructures on anodic alumina film}

Figure 4 (a) shows the FE-SEM images of microstructures fabricated on anodic alumina film by using a $5 \mathrm{~mW}$ laser beam. The thickness of the anodic alumina film is $32 \mu \mathrm{m}$, obtained after anodizing 


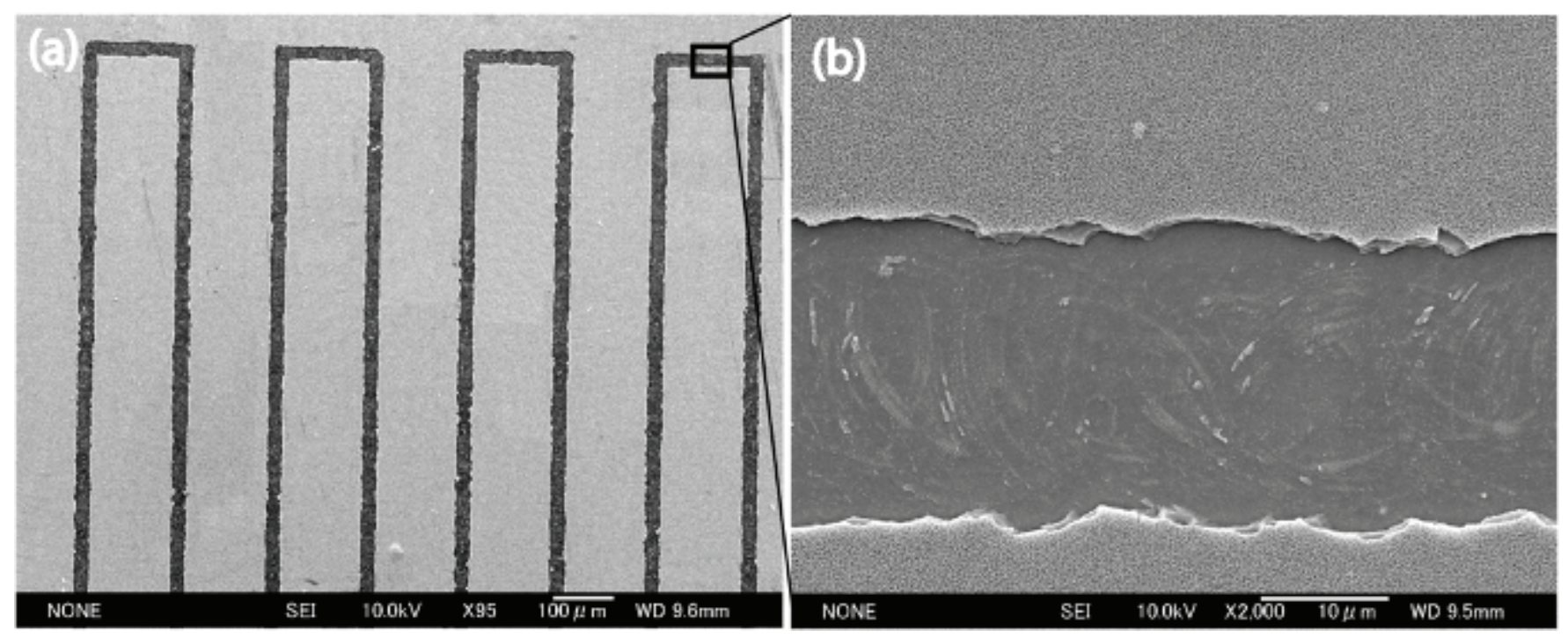

Figure 4: FE-SEM images of microstructures formed on $32 \mu \mathrm{m}$ thick anodic alumina film by removing surface layers of the film by low power laser beam $(5 \mathrm{~mW})$.

aluminum for 2 hours followed by coloring and pore-sealing. The microstructure has a uniform and smooth shape across the laser machined area. The depth of the structure is about $1.2 \mu \mathrm{m}$ with good edge definition. Figure 4(b) shows a magnified view of a portion of the microstructure, indicating that the upper layers (i.e. outer acicular crystalline layer and intermediate compact layer) are precisely removed from the surface. Figure 5 shows the variation in depth of microstructures with laser scanning speeds at 4, 6, 8 and $10 \mathrm{~mW}$ laser power beams on $64 \mu \mathrm{m}$ thick anodic alumina film. It can be seen that, at a laser power of $4 \mathrm{~mW}$ the microstructure is about $1.2 \mu \mathrm{m}$ deep at all scanning speeds. However, the structures fabricated with $6 \mathrm{~mW}$ at higher scanning speeds shows the depth of about $1.2 \mu \mathrm{m}$ but at low scanning speeds the groove is much deeper. In general, the depth increases with decreasing scanning speed as well as increasing laser power.

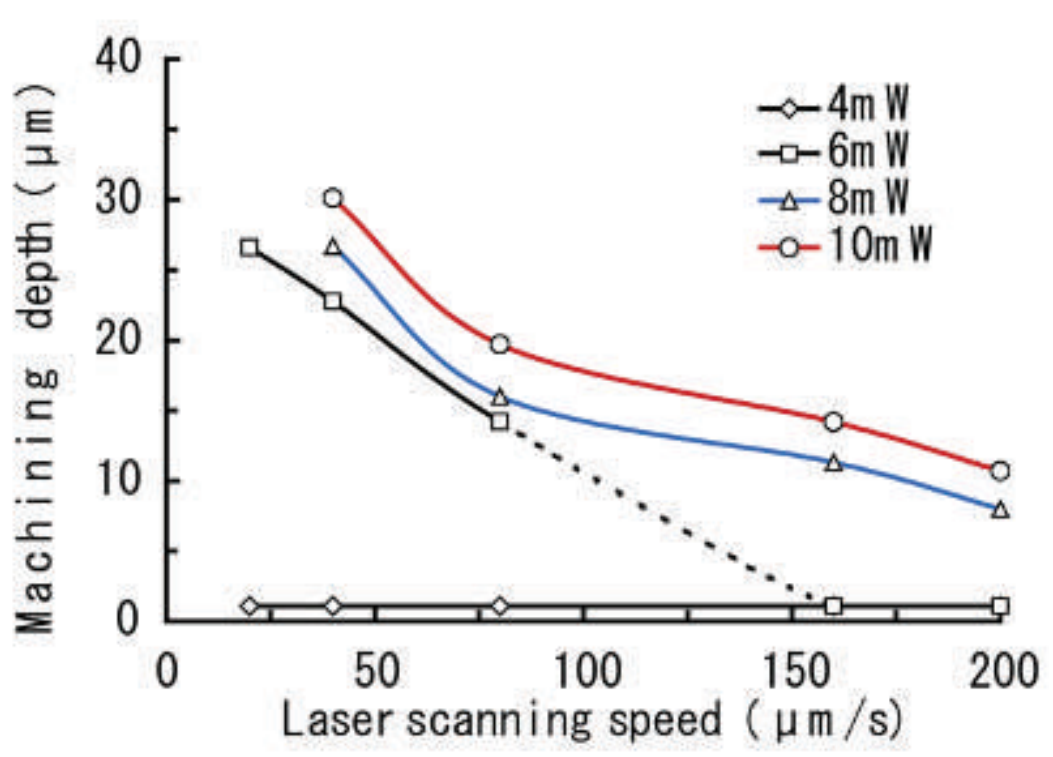

Figure 5: Variation in the depth of microstructures formed on anodic alumina film with laser scanning speed at different laser powers. porous film. However, applying higher laser power, 6 or $8 \mathrm{~mW}$, the $32 \mu \mathrm{m}$ thick anodic alumina film either breaks or forms discontinuous structures of irregular depth. Therefore, the thickness of the anodic alumina film must be increased to get deeper microstructures with good shape. It is obvious that the higher laser power beam with low scanning speed deposits much energy at the irradiated part, which re 


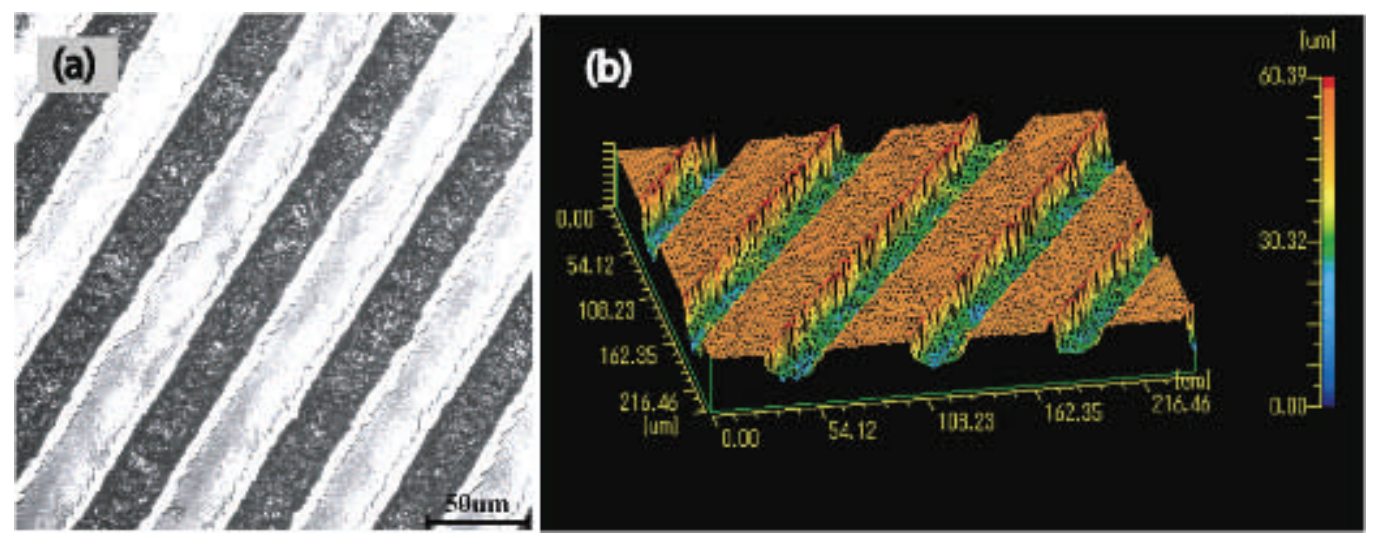

Figure 6: CSLM images of microchannels formed on $64 \mu m$ thick anodic alumina film machined by $6 \mathrm{~mW}$ laser beam with a scanning speed of $20 \mu \mathrm{m} / \mathrm{s}$. (a) Surface view of the channels; (b) 3-D height image of the channels.

sults in deeper microstructures.

The shape and size of the deeper microstructures formed by the laser micromachining is highly uniform and re-producible, as shown in Fig. 6. Figure 6 (a) shows a CSLM image of closely spaced microchannels fabricated with $6 \mathrm{~mW}$ laser power and a scanning speed of $20 \mu \mathrm{m} / \mathrm{s}$ on $64 \mu \mathrm{m}$ thick anodic alumina film, the channels were fabricated separately one by one, on the film under similar conditions. Similarly, Fig. 6 (b) shows a three-dimensional topographic image of the surface of Fig. 6 (a). The microchannels have uniform inner surfaces with similar channel depths of about $26 \mu \mathrm{m}$ with very good reproducibility. It was found that, machining under the same experimental conditions always results in similar dimensions of the microstructures.

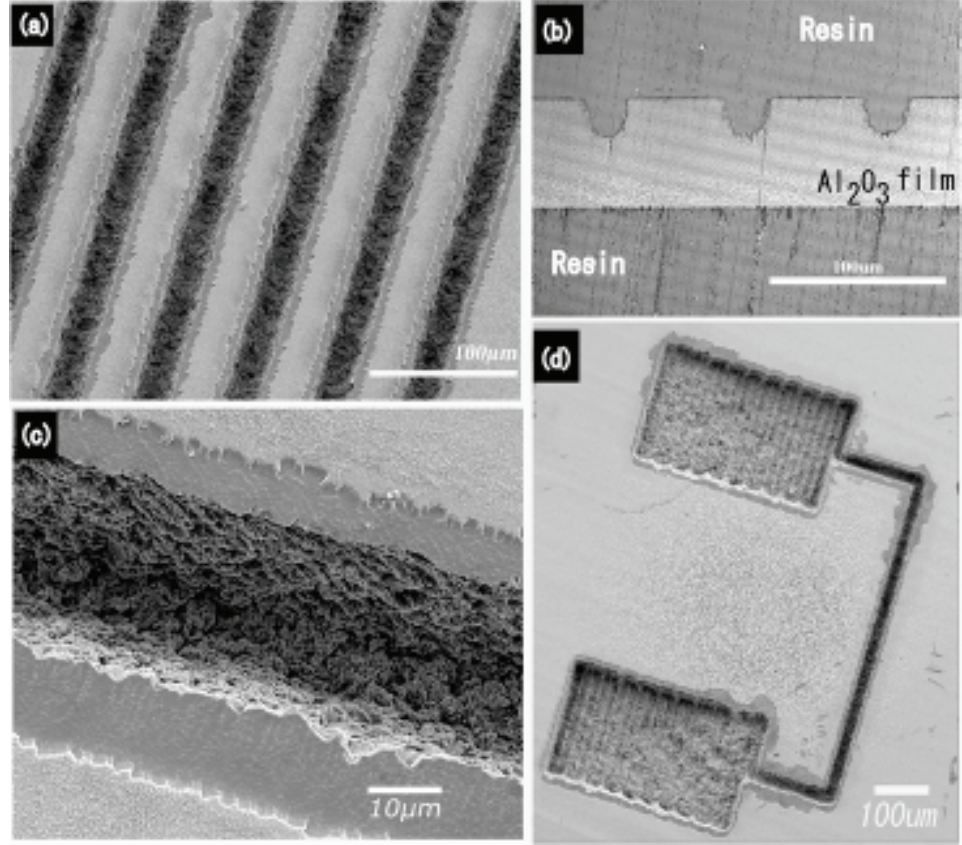

Figure 7: Images of microstructures fabricated on $64 \mu \mathrm{m}$ thick anodic alumina film machined by a $6 \mathrm{~mW}$ laser beam with scanning speed of $20 \mu \mathrm{m} / \mathrm{s}$. Here the depths of the channels are about 26um, and the depth of the chamber is about $35 \mu m$. (a) Closely spaced microchannels; (b) magnified cross-sectional view of the channels in (a). (c) Magnified view of a microchannel. (d) Micro-chambers connected with a microchannel.
Figure 7 shows microstructures fabricated on anodic alumina film by laser micromachining. Here, the $64 \mu \mathrm{m}$ thick film was micro-machined by a $6 \mathrm{~mW}$ laser power beam with a scanning speed of $20 \mu \mathrm{m} / \mathrm{s}$. Fig. 7(a) shows deep microchannels on the film having similar dimensions. Fig. 7 (b) shows the CSLM image of cross-sections of microchannels, taken after embedding the film in resin. The channels are about $26 \mu \mathrm{m}$ deep and machined precisely from the film surface. A magnified view of a channel is shown in Fig 7(c). It can be seen that, the surface layers are detached irregularly, but selectively alongside the channels. No damage or cracks were found in the cross-section. Similarly, microchambers can also be fabricated on the anodic alumina film by overlapping laser machined areas, as shown in Fig. 7 (d). Here, the two micro-chambers are connected with a microchannel. The microchambers are slightly deeper than the channels ( $\sim 32-$ $35 \mu \mathrm{m})$, which is due to the overlapping of the laser machined area. The microstructures formed on the anodic alumina film by laser micromachining are of good shape and free from the undesired cracks that are observed when machining the film attached to the aluminum substrate [14]. It is hoped that the microstructures on anodic alumina film, detached from the substrate, can be used to fabricate a range of micro-sized devices such as micro-fluidic devices, high temperature micro-devices, and also be widely applicable to the microelectronics industry. 


\section{Conclusions}

Laser micromachining on porous anodic alumina film after removed from the substrate can be carried out after coloring and pore-sealing of the film. Mainly, there are two different modes of laser removal to fabricate microstructures on the film, one by removing the surface layer of the film, and another by ablating the bulk porous layer of the film. Removal of the aluminum substrate from the anodic alumina film, followed by laser micromachining greatly improves the quality of the microstructures. Microstructures from about $1 \mu \mathrm{m}$ to several tens of micrometers depth can be achieved by choosing appropriate laser power, laser scanning speed, and film thickness. The microstructures have good edge definition and uniformity.

\section{Acknowledgements}

The research was partially supported by a Grant-in-Aid from the Ministry of Education, Culture, Sports, Science and Technology, Japan, and Light Metal Education Foundation of Japan.

\section{References}

1. P. Rai-Choudhury (ed.): Handbook of Microlithography, Micromachining, and Microfabrication. Volume 2: Micromachining and Microfabrication (SPIE-International Society for Optical Engine 1997)

2. M.J. Madou: Fundamentals of Microfabrication: The Science of Miniaturization, second ed., (CRC Press, Boca Raton, FL 2001)

3. J.M. Bustillo, R.T. Howe, R.S. Muller: Proceedings of the IEEE 86, 8, (1998)

4. M. Geissler, Y. Xia: Adv. Mater. 16, 1249 (2004)

5. M.C. Wanke, O. Lehmann, K. Muller, Q. Wen, M. Stuke: Science 275, 1284 (1997)

6. A. Pique, D.B. Chrisey: Direct-write Technologies for Rapid Prototyping Applications (Academic Press 2002)

7. C.A. Lee, N. Tsukada, T. Nakao, T. Higuchi: Appl. Phys. A 86, 201 (2007)

8. M. Stuke, K. Mueller, T. Mueller, K. Williams, R. Oliver, D.A.A. Ohlberg, G. Fuhr, R.S. Williams: MRS Bulletin 32 , $32(2007)$

9. E.C. Harvey, P.T. Rumsby: Proc. SPIE 3223, 26-33 (1997)

10. N.I. Mukhurov, G.I. Efremov, I.F. Kotova: Proc. Of the Technical Digest of IVMC'97, Kyongju, Korea (1997)

11. K.I. Delendik, and O.L. Voitik, Proc. of SPIE, MICRO/MEMS 2001, 4592 (2001)

12. T. Kikuchi, M. Sakairi, H. Takahashi, J. Electrochem. Soc. 150, C 567 (2003)

13. H. Jha, T. Kikuchi, M. Sakairi, H. Takahashi: Electrochem. Commun. 7, 1596 (2007)

14. H. Jha, T. Kikuchi, M. Sakairi, H. Takahashi, Electrochim. Acta 52, 14, 4724 (2007)

15. K. Wefers, Aluminium 49, 9, 622 (1973)

16. V. Lopez, M.J. Bartolome, E. Escudero, E. Otero, J.A. Gonzales, J Electrochem. Soc. 153, 3, B75 (2006)

17. S.I. Anisimov, B.S. Luk'yanchuk, Physics-Uspekhi 45, 3, 293 (2002)

18. A.J. Pedraza, Nucl. Instr. and Meth. In Phys. Res. B 141, 709 (1998)

19. C. Korner, R. Mayerhofer, M. Hartmann, H.W. Bergmann, Appl. Phys. A 63, 123 (1996)

20. B.N. Chichkov, C. Momma, S. Nolte, F. von Alvensleben, A. Tunnermann, Appl. Phys. A 63, 109 (1996) 